Referencia al citar este artículo:

Roy, S. (2015). Le cataclysme palestinien: les conséquences économiques du conflit israélo-palestinien. Revista TEMAS, 3(9), 31 - 46.

\title{
Le cataclysme palestinien: les conséquences économiques du conflit israélo-palestinien ${ }^{1}$
}

Stephanie Roy ${ }^{2}$

Recibido : 30/05/2015

Aprobado : 25/06/2015

\section{Résumé}

Le conflit israélo-palestinien est un litige militaire, politique et social qui perdure depuis plus de 60 ans. Les conditions économiques et sociales d'un pays en guerre sont sans nul doute affectées par les différents conflits armés, et c'est notamment le cas de la Palestine. Cet article porte donc sur l'état actuel de la Palestine et cherche à démontrer que l'économie de ce territoire est en lien direct avec les affrontements et le litige qui persiste entre les deux entités territoriales. Ce texte est basé sur une réflexion critique sur l'emprise politique et sociale d'Israël sur la Palestine à partir des approches théoriques avancées par la discipline des Relations Internationales.

\section{Mots-clés}

Résolution 242 de l'ONU, conséquences économiques, territoire palestinien.

\section{The Palestinian cataclysm: The economic consequences of the Israeli-Palestinian conflict}

\begin{abstract}
The Israeli-Palestinian conflict is a military, political and social disagreement that has now been going on for more than 60 years. The economic and social consequences of a country in a context of war are undoubtedly affected by armed conflicts, and this is the case of the Palestinian occupied territories. This article, as things stands, examines the current situation of Palestine and seeks to show that the economic conditions of the occupied territories are directly linked to the conflict between both territorial entities. Drawing upon International Relations Theories, this text is based on a critical reflection with regards to the political and social control that Israel exercises over Palestine.
\end{abstract}

\section{Keywords}

NATO Resolution 242, Economic Consequences, Palestinian Occupied Territories.

\footnotetext{
1 Article de réflexion.

2 Baccalauréat en Sciences Politiques/Relations Internationales de l'Université du Québec à Montréal (UDAM), Canada. Elle travaille actuellement pour Opération Enfant Soleil. Courrier électronique: stephanie.royy@gmail.com
} 


\section{El cataclismo palestino: las consecuencias económicas del conflicto palestino-israelí}

\section{Resumen}

El conflicto palestino-israelí es una disputa militar, política y social que ha persistido por más de 60 años. Las condiciones sociales y económicas de un país en guerra, sin duda, están afectadas por los diferentes conflictos armados, y esto se denota particularmente en el caso de Palestina. Por tanto, este artículo se centra en la situación actual de Palestina y busca demostrar que la economía de Palestina está directamente vinculada con los enfrentamientos y los conflictos entre las dos entidades territoriales. Basado en las premisas teóricas de las relaciones internacionales, este texto es una reflexión teórica sobre el control político y social de Israel sobre Palestina.

\section{Palabras clave}

Resolución 242 de la ONU, consecuencias económicas, territorio palestino.

Un peuple sans terre allait pouvoir s'installer sur une terre sans peuple.

Theodor Hezl. L'État juif (1896)

1948. L'État d'Israël est créé mais non pas reconnu par tous. C'est le début d'une guerre sans merci entre Arabes de Palestine et Juifs d'Israël. N'étant pas sans savoir que plusieurs causes expliquent le conflit israélo-palestinien et qu'il perdure depuis des décennies, il nous est toutefois possible d'expliquer d'où proviennent les tensions qui imbriquent le conflit. II s'agit à prime abord d'une question de territoire. Le conflit tel qu'on le connaît tire en effet son origine du fait que Palestiniens et Israéliens s'entredéchirent pour occuper le même territoire et revendiquent la propriété des villes saintes de Jérusalem et Bethléem. La reconnaissance des territoires s'entremêlent avec une question identitaire directement reliée à ceux-ci. Des études importantes sur le pouvoir symbolique, notamment par Pierre Bourdieu, sociologue, anthropologue et philosophe français, dénotent que les "structures symboliques" (religion, langue) sont des " structures structurantes d'une société" (Bourdieu, 1977). En soi, la langue et la religion sont des facteurs de construction du monde objectif. Israël en est un exemple notoire : la construction d'un État à partir de la religion et de la langue en vue de la formation d'une nation sioniste. L'identité du peuple juif d'Israël est en lien direct avec les structures symboliques que sont la langue et la religion; I'hébreu et le judaïsme. Quant aux Palestiniens; une société également construite autour de la langue arabe et la cosmovision rattachée à l'Islam. De là, un désir de former deux sociétés distinctes, mais sur un même territoire.

C'est peu de temps après la fin de la Deuxième Guerre mondiale que les Nations Unies tenteront de partager le territoire entre ces deux peuples, déjà persécutés des guerres de l'époque de I'Empire ottoman. Les conflits et les tensions se poursuivent à travers plusieurs guerres marquantes de I'histoire. Parmi les plus célèbres malgré elles; la Crise du Canal de Suez (1956), la Guerre des Six Jours (1967), Début de la Première Intifada (1987), Guerre du Golfe (1991), début de la Seconde Intifada (2000), bombardements récents sur la Bande de Gaza (2014), etc. Le territoire qu'occupent aujourd'hui Israël, la Cisjordanie et la bande de Gaza figure parmi les régions les plus instables du Proche-Orient. Ces conflits qui perdurent et qui semblent être sans fin ont des conséquences colossales et démesurées sur la population tant israélienne que palestinienne. On compte d'ailleurs plus de 700000 exi- 
lés palestiniens lors de la "nakba»" de 1948 "et maintenant plus de 4 millions de Palestiniens déplacés en raison des conflits" (Maltais, 2012). La Palestine est aujourd'hui ravagée et meurtrie par des décennies de guerres sanglantes et son économie n'en est pas moins touchée, les conflits militaires ayant des liens directs avec cette dernière. L'impossibilité d'exporter ou d'importer des biens et matériels crée un ralentissement tant sur le plan alimentaire que social. Un impact direct sur la production amène donc des répercussions directes sur la consommation et le commerce. Les entreprises et commerces ne pouvant recevoir le matériel et les services nécessaires pour fonctionner, les travailleurs ne peuvent donc exercer leurs métiers; on dénote donc nécessairement une baisse des revenus par personne et une stagnation dans toutes les sphères d'activités comme l'agriculture, la pêche et le transport (BBC Mundo, 2014).

En ce sens, cet article porte sur l'économie palestinienne et les conséquences du conflit israélo-palestinien depuis l'indépendance d'Israël de 1948. Les enjeux entourant les débats politiques et territoriaux en Palestine sont plutôt méconnus en Amérique et les récents événements de 2014 à Gaza ont montré au monde entier, une fois de plus, que les tensions entre Israël et Palestine perdurent et qu'elles affectent directement les populations. II est donc pertinent de se questionner aujourd'hui sur la situation de la Palestine, et cet article a pour objectif d'explorer sa situation actuelle à partir d'une perspective économique en donnant un vif et très bref aperçu du conflit depuis 1948. Cet article de réflexion sera analysé et rédigé à partir d'un cadre théorique d'Études en Relations Internationales et la méthode préconisée es la recherche bibliographique.

3 La nakba (تبكن) est le terme que l'on a donné à l'exode des palestiniens au cours de la guerre contre Israël de 1948.
Pour se faire, il est nécessaire d'interroger les liens entre le conflit israélo-palestinien depuis l'indépendance d'Israël en 1948 et l'économie palestinienne qui contribue à exacerber les tensions entre les deux peuples. Je propose d'abord un bref contexte historique sur le conflit depuis 1948 et son impact sur la situation palestinienne. Je tenterai ensuite d'établir un lien direct entre le conflit, notamment l'échec des accords d'Oslo, et l'économie de la Palestine aujourd'hui à travers la théorie d'Alain Gresh (2007), auteur reconnu et spécialiste des dynamiques socio-politiques au Moyen-Orient. Finalement, je relaterai les actions internationales possibles dans le cadre de la résolution de conflit de par le concept de sécurité globale et je tenterai d'exhiber l'avenir économique de la $\mathrm{Pa}$ lestine dans les années à venir selon une analyse personnelle.

\section{Une question d'identité}

L'histoire nous a démontré que dès les années 1920 les peuples juifs sont chassés d'un peu partout en Europe : «Jusqu'en 1947, de façon exponentielle, la population juive de Palestine augmente, sous l'effet de discriminations et d'intolérances croissantes en Europe, jusqu'au génocide de la Seconde Guerre mondiale perpétré par le régime nazi allemand [...]" (Crescenzi, 2007, p. 24). La question d'identité et du territoire est donc au cœur du conflit israélo-palestinien.

Edward Saïd a démontré que la pensée binaire, du "nous" et de "l'autre», Occidentaux/Orientaux ne peut exister, les entités et les cultures étant trop dépendantes les unes des autres pour que l'on puisse les séparer d'une manière radicale (Saïd, 2005). L'auteur argumente qu' «Un groupe n'est pas une entité naturelle, décidée par Dieu, mais un objet construit, manufacturé, voire dans certains cas inventé, avec en arrière-plan une histoire de luttes et de conquêtes qu'il est parfois 
nécessaire de représenter» (Saïd, 1994). Cette théorie est extrêmement pertinente dans la compréhension du conflit identitaire entre Israël et Palestiniens: deux peuples, deux sociétés qui se veulent différentes de par la construction de leur identité revendiquant le même territoire.

Cet article ne tente pas de démontrer et de questionner pourquoi tant d'austérité a été portée contre le peuple palestinien et le peuple juif, ni d'ailleurs d'expliquer le comportement et les politiques actuelles d'Israël, mais plutôt de démontrer que la question d'identité de la Palestine, et d'Israël, par le fait même, de par les éléments mentionnés préalablement, constitue un enjeu majeur face à leur désir d'appartenance et revendication du territoire.

\section{Le conflit de 1948}

À la fin de la Deuxième Guerre mondiale, ce sont des milliers de survivants juifs des camps de la mort qui sont ramenés vers un seul et même endroit afin de former des colonies juives: ils sont relocalisés dans une partie d'Israël que I'on connait aujourd'hui. Ils sont d'ailleurs conduits vers des terres cultivables, et dès lors, on dénote un déséquilibre flagrant entre terres arabes et terres juives:

Ainsi, $57 \%$ du territoire total, $80 \%$ des terres céréalières et $40 \%$ de l'industrie de la Palestine sont récupérés par la communauté juive, ne composant pourtant que le tiers de la population totale et n'ayant que $7 \%$ de la propriété foncière. (Crescenzi, 2007, p. 30)

C'est ensuite au printemps 1948 que la première guerre israélo-palestinienne éclate. De par le désir des autres pays arabes de s'approprier d'une partie du territoire, le Liban et l'Égypte sont égalent impliqués sur la ligne de front. Ce conflit est un point culminant pour expliquer la situation actuelle des Palestiniens car c'est au printemps de cette année-là que plusieurs Palestiniens vivant dans des villes arabes de l'État israélien sont chassés et forcés de quitter puisque la région passe dès lors aux mains des Israéliens. Certains endroits sont mêmes désertés vu les massacres occasionnés par la guerre déclenchée un peu plus tôt: ce sont ces exodes que l'on appelle "nakba». Par des actes organisés dont par le Plan Dalet (Crescenzi, 2007, p. 33), le gouvernement israélien expulse, liquide les terres, les civils et militaires palestiniens. De par ce conflit qui perdure jusqu'au début de I'an 1949, le territoire palestinien s'est vu restreint considérablement comparativement au territoire alloué lors du partage du territoire par les Nations Unies.

II faut toutefois comprendre qu'lsraël n'est pas l'unique cause des problèmes de la Palestine de l'époque et d'aujourd'hui d'ailleurs. Tel-Aviv a été bien plus d'une fois en conflit avec ses voisins arabes. Non pas seulement la Palestine, mais l'Égypte, le Liban tel que mentionné précédemment mais également la Syrie et la Jordanie, lors de la Guerre des Six-Jours de 1967, qu'elle perd à l'issue de la guerre "toute la péninsule du Sinaï, la Cisjordanie, et la Syrie se voit défaire du plateau du Golan» (Crescenzi, 2007, p. 43).

Malgré les conflits, les Palestiniens de la Cisjordanie avaient réussi à développer la société organisée, une certaine structure d'éducation avait pu être développée ainsi que plusieurs installations de modernisation et d'urbanisation de la société avaient été mis en branle. En considérant que la population était sous le contrôle jordanien, avant la Guerre de Six-Jours, c'est un avancement notable pour les Palestiniens qui avaient vécu les martyres et atrocités de la guerre de 1948. Le contrôle israélien sur la Cisjordanie ramène cependant la population vers un état précaire et défit les possibilités d'avancement des Palestiniens. L'Organisation pour la 
Libération de la Palestine 4 (OLP) fondée en 1964 pour représenter les intérêts palestiniens, prend de l'importance et acquiert du pouvoir et de la notoriété au sein de la population palestinienne de par son désir de revendiquer les droits des arabes face aux Israéliens mais surtout pour sa haine envers l'État sioniste. Elle en devient presque une institution politique qui gagne en popularité et qui a maintenant à son bord, un appareil militaire substantiel, non pas comparable à l'armée israélienne, mais devient un pilier marquant pour les palestiniens à l'époque. C'est toutefois un désir de pouvoir et d'expansion important de l'OLP et de ses alliés dans le Golfe qui poussera les Palestiniens encore une fois à être en conflit avec certaines populations du monde arabe, étant pris dans des conflits identitaires et de territoire, ayant occupés les terres de l'Égypte de Nasser par exemple. C'est avec les accords d'Oslo dans les années 1990 que la communauté internationale se positionne de nouveau en venant au même résultat. Pour avancer dans le processus de paix entre les deux entités territoriales, Gesh insiste que:

C'est par une négociation d'ensemble fondée sur un retrait des troupes israéliennes des territoires arabes occupés en 1967, y compris la partie de Jérusalem et le Golan syrien, le tracé de frontières entre l'État palestinien et Israël, et une solution juste et acceptable pour les réfugiés palestiniens. $(2007$, p. 23 )

Mais Tel-Aviv et plusieurs parties de I'opposition n'en démordent pas et malgré les recommandations des Nations Unies et de la communauté internationale, ils s'opposent fermement aux accords $d^{\prime}$ Oslo et penchent littéralement pour un renforcement, un élargissement et un développement accru de la communauté sioniste (Gresh, 2007, p. 29). Les États-

\footnotetext{
4 Organisation de Libération de la Palestine (OLP): Organisation politique et paramilitaire palestinienne fondée en 1964 pour représenter les intérêts palestiniens.
}

Unis, un acteur important dans le conflit depuis plus de 60 ans, et le gouvernement d'Obama ont d'ailleurs démontré une approche plus rigide quant à son allié historique qu'est Israël, toujours dans une optique de résolution de conflit.

Ce très bref aperçu historique nous permet de comprendre les éléments précurseurs de la géopolitique palestinienne actuelle. D'abord meurtrie et bouleversée par les premiers affronts avec Israël vu le désir d'expansion et de "purification" de l'État sioniste, elle s'est vue déchirée par ses désirs politiques de vengeance et de reconquête des territoires face à I'«ennemi». N'étant cependant pas le seul territoire arabe ayant été victime de ses propres conflits internes et envers ses alliés, la Palestine en reste toutefois grandement affectée, et c'est ce que nous tenterons de démontrer tout au long de cet article en balayant un portrait de la vie actuelle palestinienne et de sa situation économique.

Je suis consciente que divers éléments de l'histoire de la Palestine et de I'OLP notamment dans les années 1970 ne seront pas survolés ni étudiés dans cet article qui ne prétend d'ailleurs pas être en mesure de rendre compte de toutes les causes du conflit ni de pouvoir faire un portrait exhaustif des guerres qui ont touchées la Palestine durant le $X X^{\mathrm{e}}$ siècle. Cependant, je désire plutôt tenter de dessiner quelques causes qui ont amené la Palestine à la situation précaire que l'on connait aujourd'hui et d'ainsi mieux comprendre le conflit israélo-palestinien qui perdure depuis des décennies. Judith Butler a d'ailleurs élaboré un concept intéressant, celui de la précarité: "Cette condition générale permet d'aborder la question des conditions rendant une vie viable ainsi que les manières $d$ 'améliorer les vies et de lutter contre la précarité différentielle et spécifique.» Ce concept vient en appui au fait qu'il existe une certaine précarité des peuples, tant au niveau économique 
que social et que certains moyens doivent être entrepris afin de contrer cet état de précarité (Butler, 2010). Traiter et analyser ce sujet, soit l'état de la Palestine et à sa sphère économique est donc pertinent, dans un monde où les relations et le développement sont basés de plus en plus sur l'économie d'un pays et sa position dans le système global.

\section{Oslo et les dualités administrative et politiques}

Les accords d'Oslo de 1993 revendiquaient d'abord et avant tout une autonomie palestinienne à l'intérieur des frontières dessinées par les Nations Unies en 1967. Les accords d'Oslo et de la communauté internationale avaient de bonnes bases et souhaitaient venir à bout du conflit israélo-palestinien, toujours dans l'optique d'une paix entre les deux peuples. On réclame alors que les deux partis reconnaissent les droits politiques et administratifs de l'autre. On somme donc un retrait des troupes israéliennes sur Gaza et sur la région de Jéricho et un transfert des compétences notamment au niveau des affaires sociales, de l'éducation et de la taxation aux Palestiniens et non plus dictés par le gouvernement de Tel-Aviv (Gresh, 2007, p. 29). Une photo célèbre de l'époque relate l'accord des deux parties avec à la tête de I'OLP, Yasser Arafat et du Président israélien de l'époque, Yitzhak Rabin au côté de Bill Clinton. Une photo qui fera très certainement le tour du monde où les deux hommes se serrent la main avec à l'arrière un Clinton souriant et heureux de pouvoir participer à ce moment. Malheureusement pour les deux parties, les sourires ne sont pas restés dessinés bien longtemps.

Le partage des compétences n'est jamais chose simple. Plusieurs exemples au niveau mondial peuvent le démontrer, toutefois à très petite échelle, si l'on considère l'état actuel des relations entre israéliens et palestiniens. On parle donc, avec ce partage des compétences, de dualité. Bien souvent politique, mais à bien des égards, elle en reflète les conséquences sur l'économie, l'éducation et bien d'autres sphères d'activités pour les palestiniens : "Cette superposition et l'ambiguïté des demandes israéliennes ont aussi nourri la méfiance des Palestiniens et produit l'idée qu'il existe un plan machiavélique de conquête concertée du territoire palestinien sous couvert du processus de paix." (Debié et Fouet, 2001, p. 237) Cette dualité fait en sorte que vraisemblablement les palestiniens n'ont pas encore le droit et le pouvoir d'être régis par la Palestine; Israël en est encore en tout point responsable et maître des relations et décisions. L'inquiétude des palestiniens est fondée sur le principe de renforcement et d'élargissement qu'lsraël n'a cessé de revendiquer depuis des décennies. Les mêmes désirs et intérêts par d'autres moyens cette fois. Le jeu politique cache bien souvent les réelles intentions des partis concernés.

De ce fait, on ne peut réellement déceler, même d'un point de vue historique si Israël ou si d'ailleurs n'importe quel pays agit en fonction de ses propres intérêts ou pour l'entraide et le maintien des bonnes relations internationales. C'est là le principal enjeu des Relations Internationales. Toujours méfiants, les palestiniens de la bande de Gaza tentent donc de vivre, pourrait-on dire de survivre à travers les revendications et interdictions du géant israélien qui maintient une main de fer sur la Palestine et son économie:

" [...] Ils [Palestiniens] n'en contrôlent pas vraiment les dynamiques foncières, leurs projets d'infrastructures, les entrées et les sorties [...] L'organisation future de leur «territoire» ne leur appartient pas (encore) et ils ne savent pas ce que les Israéliens projettent à long terme" (Debié et Fouet, 2001, p. 238). 
La fin de l'occupation militaire de Tel-Aviv n'amène donc pas directement au processus de paix. Israël, exerçant encore une forte pression sur différents niveaux, autres maintenant que l'aspect militaire, ne permet pas aux Palestiniens de s'épanouir dans une société nouvelle, étant toujours sous l'emprise israélienne d'un côté comme de l'autre. Et la situation continue d'être ainsi même sous les yeux de la communauté internationale. Ce sujet sera d'ailleurs élaboré un peu plus tard.

\section{Un avenir pour la palestine?}

L'histoire nous a toutefois démontré que malgré les recommandations et interdictions de la communauté internationale, les territoires occupés par la Palestine seront accompagnés d'une série de redéploiement des troupes israéliennes d'un moment à un autre, pour diverses raisons. Cependant, comme nous l'avons illustré préalablement, malgré le retrait des troupes israéliennes et l'occupation seulement symbolique des terres palestiniennes, Israël garde l'emprise sur la Palestine qu tente de se régir par elle-même et de se développer. D'abord, d'un point de vue plus géographique, les routes et barrages, les allées et venues, les entrées et les sorties du territoire de la Palestine sont régis par Tel-Aviv: "Le contrôle des flux, de la terre et l'installation des ressortissants israéliens constituent les éléments fondamentaux de la politique de domination du territoire de la Palestine." (Debié et Fouet, 2001, p. 238) On peut également parler de l'octroi des terres aux agriculteurs qui est également régit par le gouvernement israélien. Tout doit être perçu et revu par l'État sioniste. On entendra souvent parler de la zone $C$, qui constitue en fait les deux tiers de la Cisjordanie et un tiers de Gaza (Debié et Fouet, 2001, p. 238). Les frontières, étant toujours matière à conflit dans le cadre des affrontements israélo-palestinien, se- ront encore une fois mises de l'avant avec I'obtention de la zone $\mathrm{C}^{5}$ des Israéliens.

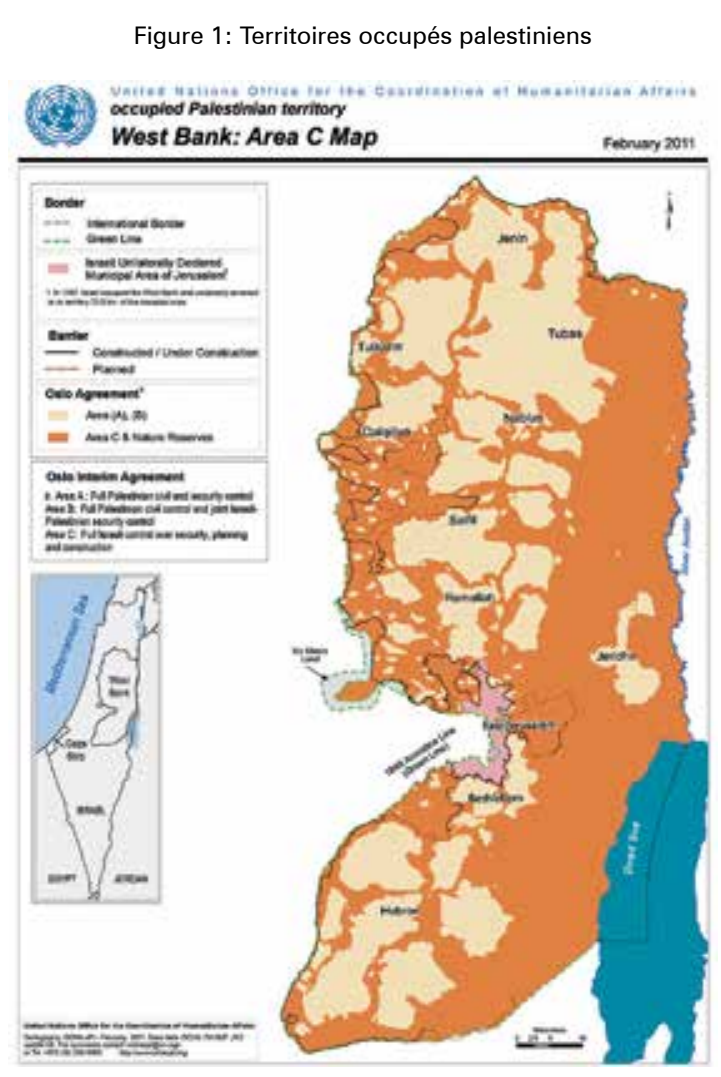

Source: Bureau des Nations Unies pour la coordination des affaires humanitaires, Territoires occupés palestiniens, https://www.ochaopt.org/mapstopic. aspx id $=109$ \&page $=1$

Ces frontières n'étant visiblement pas marqués au sol ou bien définies et reconnues par les deux partis; Israël prendra avantage avec l'appartenance de ces terres et refusera dans plusieurs cas, I'obtention de certains lots de terres aux Palestiniens, ou le droit à l'agriculture sur certaines parcelles de terres. Il en sera de même pour d'autres projets routiers ou développement industriel et urbain. La Palestine n'a donc pas dans tous les cas le pouvoir de décider de son sort.

$D^{\prime}$ un point de vue du droit international, on parle ici d'ingérence et du droit $d$ 'ingérence qui n'est aucunement soutenu par la communauté internationale

5 Une zone du territoire (notamment une partie de la Cisjordanie qui est découpée par le zonage) où Israël exerce un contrôle militaire et civil total. La zone est habitée par 150000 Palestiniens et 325000 colons israéliens illégaux. 
dans d'autres cas du reste du monde. Pourquoi donc Israël peut-il déferler son pouvoir sur la Palestine? De quel point de vue du droit international permet-on une telle ingérence? Comment en est-on donc venu à décimer la zone $\mathrm{C}$ comme appartenant à Israël?

Concernant la situation actuelle de la Palestine, en 2015, rien n'a encore changé du point de vue de l'essor de l'économie palestinienne. Les terres sont toujours sous contrôle israélien et les Palestiniens sont toujours forcés à vivre les uns sur les autres dans le petit fragment de territoire qu'est appelé la bande de Gaza. Les seules parcelles de terrain où la Palestine est "maître» de son territoire sont supposées être les zones $A$ et $\mathrm{B}^{6}$.

Figure 2: Les différentes zones du territoire

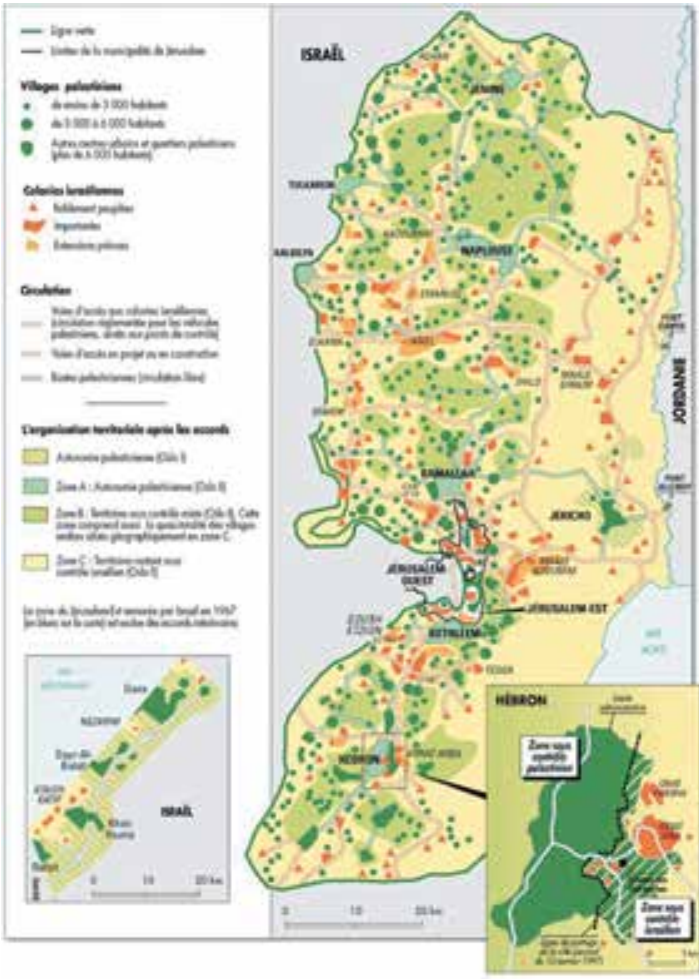

Toutefois, Israël exerce encore là des pressions et maintient le contrôle sur ces terres également. Un fait intéressant est

6 Zones $A$, B et C du territoire. Source: http://www.bbc.co.uk/ mundo/noticias/2014/07/140715_gaza_palestinos_israel_vida_ cotidina mz.shtml que tout Palestinien, à Gaza ou JérusalemEst est régi par l'autorité israélienne. Cependant, dans le cas contraire, un Israélien en territoire jugé palestinien ne sera pas régi par les Autorités Palestiniennes. ${ }^{7} C^{\prime}$ est un contrôle de pouvoir dans cette dualité qu'exerce donc Israël sur la Palestine. Deux acteurs, à parts bien inégales, l'un ayant le contrôle absolu et illégitime sur l'autre.

Afin de brosser un portrait réel de la Palestine, il ne faut pas oublier les apports que les accords d'Oslo ont amenés aux Palestiniens. La situation est en fait dérisoire mais la Palestine peut tout de même s'organiser d'un point de vue juridique, municipal et au niveau des forces policières. Ils vivent désormais sous une sorte de dualité juridique, ayant, avec un comparatif très primaire de la situation, deux "niveaux» juridiques. Les Autorités Palestiniennes existent bel et bien et sont une force de l'ordre et peuvent régir et sévir dans certains domaines:

Les Palestiniens de Gaza et de Cisjordanie, surtout ceux qui travaillent en Israël, vivent désormais, et c'est là la nouveauté, la dualité juridique de leur espace "en direct» lls paient les impôts palestiniens, obéissent aux lois palestiniennes, s'acquittent de leurs obligations vis-à-vis des autorités palestiniennes, auprès désormais solliciter les autorisations, permis, licences pour l'extension de leur maison, l'immatriculation de leur nouvelle voiture, l'installation de leur parabole [...] (Debié et Fouet, 2001, p. 243).

Mais les autorisations doivent subvenir bien avant les renouvellements ou les extensions. En résumé, les Palestiniens doivent donc de se doter d'une autorisation de Tel-Aviv afin d'acquérir une maison par exemple, mais en demander un permis aux autorités palestiniennes pour

7 Entité du gouvernement ou autrement appelé forces de l'ordre qui régit les habitants arabes de Cisjordanie et des zones $A$ et $B$ de la Palestine (zones qui ont érigés de par les accords d'Oslo de 1993). 
I'extension de celle-ci. Ils peuvent sans quoi se voir restreindre cette autorisation ou ce droit, par pur et simple pouvoir discrétionnaire.

Oslo n'a toutefois pas été un échec sur toute la ligne. Bien que cette dualité existe:

[...] on a vu avec l'arrivée au pouvoir de l'Autorité palestinienne en 1994, les conseils municipaux et villageois reprendrent leur fonction administrative. Ils sont responsables de l'eau, de l'électricité, de l'enlèvement des ordures, des établissements scolaires, du contrôle de la planification et de la construction, de l'entretien et de la construction des routes, du contrôle des marchés publics." (Debié et Fouet, 2001 , p. 245).

Même s'il s'agit d'un contrôle normal et présent dans chaque État du reste du monde, il n'en reste pas moins que c'est en lien direct avec les accords d'Oslo que la Palestine a pu reprendre le contrôle de ces compétences et activités au sein la société. C'est un point notable qu'il ne faut pas oublier et enlever à Oslo.

L'opinion publique critiquera Oslo comme étant un échec des accords de paix dans le conflit, mais cet avancement est notoire et amène plus de flexibilité aux palestiniens dans un monde où la modernité frappe de tous les côtés. Bien que les autorisations doivent tout de même être faites à Israël, à petite échelle, on peut considérer le retour des compétences aux Autorités Palestiniennes comme un avancement important dans le conflit. La Palestine peut enfin sortir du mode de colonie à laquelle elle était confinée depuis bien des années. Avec l'élaboration de comités et le rétablissement de la démocratie et de la représentation locale, la Palestine peut commencer à sortir de son état précaire.

Mais les Autorités ont également restructuré les frontières. Frontières qui causent depuis des décennies des conflits interminables et meurtriers, qui ont poussé à l'exode de plusieurs centaines de milliers de Palestiniens lors de la nakhba, mais également tout au long du $X X^{e}$ siècle de par l'égide de Tel-Aviv sur les arabes de Palestine. Les Autorités Palestiniennes n'ont ni repris le modèle britannique d'avant 1948, ni le modèle jordanien ou israélien mais,

[...] s'est vu dessiner une géographie virtuelle du territoire, prolongeant d'un côté le passé arabe, en élevant en gouvernorat les anciens districts jordaniens et préfigurant d'un autre avenir. Le découpage palestinien reconstitue ainsi par anticipation, au détriment des districts «israéliens» de Bethléem et de Ramallah, au gouvernement de Jérusalem, dans des limites voisines [...] (Debié et Fouet, 2001, p. 246).

On se retrouve donc face à plusieurs divisions des frontières, très différentes et revendiquées par les deux parties qui n'arrivent pas à s'entendre. D'un point de vue légal, rien n'a été approuvé, les frontières ayant été arrimées de manière à plaire aux deux partis concernés. Ces frontières ne sont ni approuvés par I'autre parti, ni définis mutuellement. On en arrive donc à la situation actuelle : la Palestine revendique une partie de son territoire comme étant Palestinien, et Israël revendique cette même parcelle de territoire comme étant sienne, dû aux définitions et élaborations différentes de ses propres frontières. De par cette dualité, découle plusieurs paliers administratifs, juridiques, sociaux et économiques :

La même famille peut avoir à traiter avec la police locale mais aussi avec les forces de sécurité spéciale, sorte de $\mathrm{FBI}$ palestinien, avec les autorités municipales élues ou nommées, avec les représentants locaux de différentes administrations nationales, avec les autorités du gouvernement, sans compter les contacts inévitables avec les auto- 
rités israéliennes [...]. (Debié et Fouet, 2001, p. 254)

L'arrivée en force des administrations palestiniennes et un pouvoir retourné à la Palestine a pris des proportions démesurées en matière d'autorité à divers paliers ; un couteau à double-tranchant diront certains. La réglementation sous différents comités et paliers de gouvernement occasionnent donc un impact direct sur les Palestiniens qui ne peuvent se défaire de l'autorité israélienne, mais qui se voit également contraints à obéir et à être régis par plusieurs paliers de l'autorité de son propre "gouvernement". Par toutes ces contraintes, on peut également voir apparaître au sein de la population palestinienne, l'invasion fiscale pour éviter de payer les taxes sur plusieurs paliers de gouvernement, des débordements de toutes sortes afin d'éviter d'être contraint aux différentes administrations, on tente d'obtenir des faveurs ou autorisations diverses par le simple fait de connaître quelqu'un ayant le pouvoir, que l'on appeIle wasta (نُس) en arabe, afin d'esquiver les nombreuses lois et réglementations des paliers de contrôle palestiniens et israéliens:

La complexité des accords et la complication des cadres et des structures administratives de la Cisjordanie conduisent donc légitimement à faire l'évolution concrète sur le terrain-les attentats pour les Israéliens, la question foncière pour les Palestiniens-le test de vérité de la volonté de la paix. (Debié et Fouet, 2001, p. 255)

\section{Le commerce}

Le retour de certains pouvoirs à l'administration palestinienne de par les accords d'Oslo n'en demeure pas moins des contraintes face à l'économie et aux relations internationales et commerciales que la Palestine pourrait avoir. D'abord, j'ai démontré que certaines compétences ont été octroyées de nouveau aux
Autorités Palestiniennes, d'un point de vue plus local et municipal, mais la main de fer d'Israël sur toutes allées et venues des Palestiniens et de ce qui entre sur le territoire palestinien restreint un éventuel commerce palestinien ainsi que des relations économiques avec le reste du monde. Les Palestiniens éprouvent des difficultés immondes à importer autant des biens et matériels que de la nourriture, devant passer par les ports israéliens et d'emblée, par le contrôle de Tel-Aviv. Le commerce en est également difficile via les exportations pour la Palestine. Elles sont extrêmement contrôlées, d'autant plus que le gouvernement israélien interdit l'importation de certains produits en Palestine.

Le commerce se résume donc pratiquement à la vente d'articles à Israël et vice-versa. Les prix sont toutefois doublés parfois même triplés lorsqu'Israël vend aux Palestiniens : "Le marché de Jérusalem-Est continue d'être fermé aux producteurs et aux agriculteurs palestiniens de Cisjordanie pour des raisons de "santé publique» et de normes." (Debié, Fouet, 2001, p. 284) Les agriculteurs et producteurs sont donc contrés à la revente à l'intérieur même de leur territoire, ne pouvant exporter à l'international, tel que mentionné précédemment. En ce qui concerne l'acquisition de produits, le citoyen palestinien en est contraint à ce qu'il peut trouver sur le marché palestinien et ce qu'il peut acquérir des israéliens. La liste des produits interdits à l'importation est longue et plutôt facétieuse de produits alimentaires et matériaux de toute sorte :

Les matériaux de construction et les matières premières ont été sur la liste des produits interdits par Israël depuis 2006, comme le ciment, I'acier et le béton. Israël a toujours pris le prétexte que ces articles pourraient être utilisés par des groupes de la résistance dans Gaza pour construire des constructions à vocation militaire. (Omer, 2014) 
Certains produits alimentaires sont également proscrits pour les Palestiniens par les Israéliens. La liste exhaustive n'a toutefois jamais été publiée, sujette à des changements, mais selon certaines sources, le chocolat, le café soluble ainsi que la confiture ont parfois figuré sur cette liste des interdits. Ces conséquences économiques sont en lien direct avec le mécontentement de la population et des travailleurs qui en souffrent inconditionnellement. Moins d'exportations et d'importations font donc une économie précaire et appauvrie, moins de commerce veut donc dire moins de travail et par le fait même moins de salariés. Aucune possibilité d'avancement économique ni de boom économique et du commerce s'arrime donc avec des salaires moindres voir des salaires quasi-inexistants; des familles sous le seuil de pauvreté et très peu pour se nourrir et subvenir à leurs besoins. L'asymétrie économique est donc un élément important. La reconnaissance de la communauté internationale est également un point à prendre en considération et qui a énormément de poids quant au développement économique de la Palestine. Israël est un État, et ce, depuis 1948. Malheureusement, la Palestine n'a pas reçu la reconnaissance internationale de tous et c'est un point culminant directement lié à ses possibilités de développement et de commerce quant au reste du monde. C'est un point pertinent à prendre en considération quant à l'élaboration de solutions pour l'avenir économique de Palestine.

La Palestine vit donc encore aujourd'hui dans une situation précaire. Certaines améliorations d'un point de vue des politiques administratives et régionales provenant des accords d'Oslo tentaient de donner une lueur d'espoir aux palestiniens, mais les isolaient toutefois du monde extérieur par l'oppression de TelAviv. Une situation économique appauvrie et désuète et d'infimes possibilités de changements vu la radicalisation d'Israël. Pour le moment, la Palestine est confinée à l'agriculture de subsistance et quelques rapports commerciaux avec Israël, mais les ouvertures et possibilités pour les travailleurs en restent au point mort, encore aujourd'hui.

Par ailleurs, les menaces non militaires sont aussi des menaces à la sécurité et des contraintes pour la paix d'un État. Tel que mentionné préalablement, Israël n'occupe plus militairement, du moins à court ou à moyen terme ces dernières années le territoire de la Palestine. Les menaces et les pressions militaires se font toutefois toujours sentir ainsi que I'occupation symbolique et les humiliations quotidiennes. Les récents bombardements de 2014 nous ont démontré la situation fragile entre les deux entités territoriales et la nette domination israélienne sur le destin de la Palestine. D'autres facteurs que l'aspect militaire par exemple, sont enclins à la sécurité de la Palestine, également expliqué plus tôt. Cette thèse néolibérale des menaces non militaires explique en tous les cas la situation des Palestiniens.

Les menaces non militaires résultent de conditions politiques, économiques, sociales ou environnementales qui mettent en cause le fonctionnement et la survie de l'État et, à l'occasion, provoquent des conflits et une violence civile qui bouleversent les conditions d'existence des populations." (David, 2006, p. 110)

Comme l'a démontré Richard Ullman, l'un des précurseurs de la théorie de la sécurité globale,

[...] la notion de dégradation" est directement liée à la sécurité. Selon lui, la sécurité, est menacée dès lors qu'une succession d'événements fait craindre, sur un court laps de temps, une diminution drastique de la qualité de vie pour la population d'un État [...] (David, 2006, p. 110). 
La Palestine en est en fait un de ces exemples. L'histoire nous a démontré à travers tous les conflits du $X X^{e}$ siècle que la sécurité de la Palestine en était menacée de par plusieurs aspects, et ce, malgré la non-occupation militaire du territoire. En soi, une privation des ressources, comme l'eau par exemple est sujet à la sécurité, toujours ici appuyant la théorie d'Ullman repris ici par David. C'est de dire que l'aspect de sécurité est maintenant revu sur plusieurs paliers et qu'une nation est enclin non pas seulement militairement par des forces de déploiements ou par des combats menés par l'armée, mais également par des pressions et litiges économiques et sociales. David relève également dans son livre un aspect important de la sécurité alimentaire applicable encore une fois à la situation économique de la Palestine :

La Commission Brandt sur le développement international identifie, en 1983, la relation qui existe entre la sécurité et le développement et avance la thèse que les divisions entre pays riches et pays pauvres vont croissant et menacent de provoquer des injustices et des famines graves. (David, 2006, p. 110)

Prenons ici, encore une fois l'aspect qui nous intéresse. Le lien entre la sécurité et le développement. II est évident que dans l'optique où la sécurité de la nation est menacée, l'État ne peut axer sur le développement, et ce, dans tous les sens du terme. Les accords entre Israéliens et Palestiniens sont bien souvent propices à des frictions et des conflits militaires en résorbent bien plus souvent qu'autrement. Les tentatives d'accord de paix ont généralement été dans I'histoire un échec total, non pas que les Accords d'Oslo en ont été, mais du moins n'ont pas réussi à faire accepter toutes les clauses du rapport et des accords aux deux partis concernés, étant deux protagonistes de force inégales. De ce fait, non pas uniquement par la menace militaire mais également par les aspects de sécurité mentionnés précédemment, la Palestine ne peut prospérer économiquement, ni d'ailleurs socialement, comme par exemple en lien avec la contrainte alimentaire. Des exportations et importations difficiles, un commerce quasi-inexistant, une agriculture de subsistance réduisent considérablement les chances des Palestiniens de se développer et de s'urbaniser.

\section{Conclusions : comment résoudre le conflit?}

Personne ne détenant la science infuse, il n'y a pas une seule solution à appliquer et non pas uniquement une seule façon de faire et de méthodes à utiliser. Comme l'écrit Gresh, « il est avant tout vital que la communauté internationale comme Israël reconnaissent le tort qui leur a été fait [en référence à la Palestine]. Leur drame mérite une reconnaissance morale» (Gresh, 2007, p. 161). De mon point de vue, Gresh a effectivement raison : dans l'optique des relations internationales et commerciales fortes entre les pays au détriment des valeurs identitaires étatiques individuelles, afin de prospérer tant économiquement, que politiquement, l'État doit voir au-delà de ces propres intérêts et abandonner les pensées du réalisme de Kenneth Waltz. Autant dans le jeu politique que pour ses propres intérêts, un État ne peut que sortir gagnant en privilégiant et développant ses échanges économiques. Dans cette optique, j'appuie favorablement sa théorie selon laquelle Israël doit reconnaître le tort qu'il a causé aux Palestiniens depuis la création de l'État juif. Les États arabes, la Palestine y compris, doivent cependant reconnaître qu'lsraël a droit à une reconnaissance de son territoire également, et parlant de ce territoire, nous faisons référence aux frontières dessinées par les Nations Unies en 1967 (Gresh, 2007, p. 161). ${ }^{8}$ Ces frontières ont été dessinées par la Résolution 242 des Nations Unies, mais

8 Zones orange sur la carte devant être attribués à la Palestine. 
non tout simplement pas été respectées. Dans l'optique d'une résolution de conflit, on doit appliquer ces frontières comme étant la séparation de deux états distincts.

Figure 3: Le conflit israélo-palestinien depuis 1967

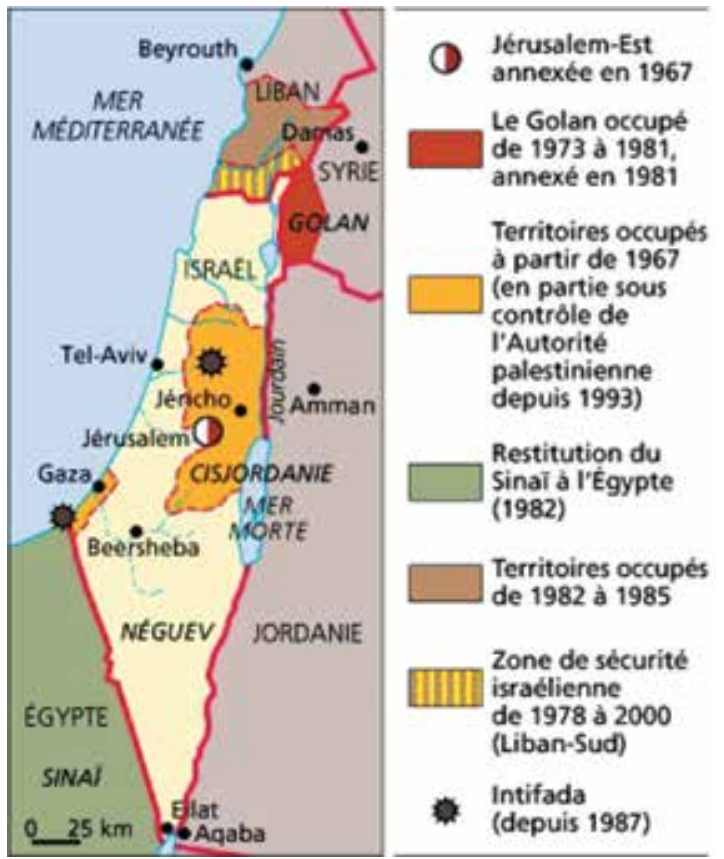

Source: Larousse, http://www.larousse.fr/encyclopedie/pays/ Isra\%C3\%ABI/125296

À ce sujet, afin de lutter contre la précarité, Butler prétend également qu'«à partir de la présomption d'égalité, on peut élaborer des politiques sociales qui cherchent à affirmer ce principe et à organiser l'aide pour des besoins matériels sur la base de l'interdépendance» (Butler, 2011). La reconnaissance de chacun doit être un des piliers de la résolution de conflit. Ensuite, on pourra développer des liens commerciaux importants de par l'interdépendance, tel que le mentionne Butler. En ce sens, Éthier argumente que les " changements qui se produisent à l'intérieur de chaque société ont une incidence sur le système international et les transformations du système international ont un impact sur la dynamique interne de chaque société» (2006, p. 51). En appui à cette théorie des néolibéraux, je suis d'avis que la société palestinienne gagnerait d'abord avec un processus de paix à bien des égards. Bien sûr, le processus vers la paix est un long périple qui tarde à se concrétiser, mais n'est pas impossible.

$D^{\prime}$ abord faudrait-il qu'lsraël laisse tomber ses élans néoréalistes et usent de diplomatie. Tel-Aviv a clairement démontré un penchant vers la pensée réaliste classique de Morgenthau (Éthier, 2007, p. 51) depuis son indépendance mais notamment depuis les accords d'Oslo de 1993. Un penchant vers ces théories qui revendiquent un État fort, dans l'optique où,

[...] la loi objective fondamentale qui gouverne les relations internationales est le fait que les États agissent toujours dans le but de défendre leurs intérêts ou leur puissance politique, qui est indépendante de l'économie, de l'éthique [...]. La puissance d'un État peut inclure toute chose qui établit et maintient le contrôle de I'homme sur I'homme.» (Éthier, 2007, p. 51)

Comme l'explique la théorie néolibérale, la prospérité d'une société, ainsi que son urbanisation par le fait-même, découle directement des relations internationales et économiques avec le reste du monde. La Palestine, pour les raisons que nous avons vues précédemment reste dans un était précaire, ne pouvant subvenir aux besoins de sa population et donc ne peut penser à organiser quelques relations commerciales que ce soit, étant brimée par Israël. Les options sont donc restreintes, lorsqu'un peuple est toujours en mode survie, et ce, depuis des décennies, il est difficile de penser acquérir une certaine émergence économique que ce soit.

Notons cependant qu' «un conflit est terminé lorsque survient un accord entre les protagonistes. Cet accord met fin à la phase violente de l'affrontement" (David, 2006 , p. 283). Les deux partis doivent donc d'abord et avant tout faire preuve de détermination et laisser de côté leurs 
désirs et volontés individuelles. II faut prendre en considération qu'on devra revoir certains aspects de nos requêtes et instances et que tout ce que l'on exige ne sera pas nécessairement mis de l'avant, devant faire compromis sur certains aspects, par exemple la coexistence et le partage d'un même territoire.

L'histoire nous a cependant démontré que le conflit israélo-palestinien ne peut malheureusement se résoudre de par une entente mutuelle. Une tierce partie doit donc être présent afin d'en venir à un accord, et par la suite à une paix mutuelle. Dans un cas de médiation, des ONG ou autres organismes internationales peuvent occuper le rôle de tierce partie. Les Nations Unies (ONU) ont d'ailleurs été bien souvent présentes aux tables de négociations. Cependant, je suis d'avis que, comme I'ONU n'a aucune autorité au-delà du pouvoir étatique, et tant Israël que la Palestine, nous ont démontré maintes et maintes fois que les décisions de l'ONU quant aux conflits n'ont pas été respectées par les protagonistes, qu'une autre tierce partie pourrait être envisagée à ce moment-ci. Des gouvernements, ou un seul État par exemple toujours dans I'optique de faire respecter les accords mis en branle pour les deux partis pourraient agir à titre de médiateur: "Inciter les partis à la conciliation, c'est-à-dire les convainquant du mérite d'une démarche diplomatique susceptible d'aboutir, ultérieurement, à un processus de négociation." (David, 2006, p. 283) Comme le stipule David dans son livre, "un conflit n'est véritablement résolu et ne cède à la paix durable que lorsque les trois niveaux sont mis à contribution: des rencontres officielles, des démarches informelles et une société» (2006, p. 283).

Enfin, le conflit ne disparaît véritablement que lorsque son contexte, sa structure, ses acteurs et ses enjeux ont été profondément transformés" (David, 2006, p. 284). D'ailleurs, tel qu'expliqué par Judith
Butler et démontré précédemment, l'état de précarité doit être dépassé. On doit non pas seulement voir l'aspect humain en premier lieu mais également voir aux besoins de la population afin de contrer la violence structurelle (Galtung, 1969). En fait, la violence est parfois indirecte, mais plutôt des actions ou des décisions politiques, sociales ou économiques qui peuvent affecter la population. Dans tous ces cas, on peut quand même parler de violence, selon Galtung, et donc on en vient à brimer les libertés des individus ou ses conditions nécessaires à la survie et à sa sécurité également. La paix marche de pair avec la transformation des conflits et puisque nous avons dépassé l'étape de préventions des affrontements depuis 1948, il faut maintenant axer sur la possibilité et les moyens pour rayer les hostilités et avoir en mire la paix durable. La Palestine ne pourra prospérer ni subvenir aux besoins de sa population si la paix durable n'est pas envisagée. La situation politique a évolué depuis Oslo, on a pu en constater les apports un peu plus tôt, cependant là n'est pas non plus la situation idéale pour les Palestiniens. II faut envisager des améliorations sociales et économiques pour le peuple, et c'est pour cette raison que des rencontres officielles doivent de nouveau être mises de l'avant, que la communauté internationale doit voir à la résolution du conflit et s'impliquer en tant que tierce partie dans ces rencontres: "La paix n'existe pas en l'absence de guerre ou en l'absence de violence; elle est atteinte seulement une fois I'harmonie entre individus et communautés rétablie.» (David, 2006, p. 285).

En s'appuyant sur cette théorie et en mettant l'accent sur une démarche néolibérale, il faut d'abord voir à la mise sur pied de résolution des problématiques, rédiger des accords et veiller ensuite à une paix durable et des ententes de coopération bipartites. Faire respecter les droits des individus, assurer une 
sécurité globale, tant au niveau humain qu'au niveau alimentaire. Johan Galtung a d'ailleurs avancé une théorie importante dans les recherches sur la paix, qui estime que selon lui, "les conditions d'une paix durable exigent que toutes les formes de violence indirecte (injustice, pauvreté, oppression) disparaissent» (David, 2006, p. 287). À travers ses études, Galtung démontre d'ailleurs un aspect différent des études traditionnelles sur la paix. La violence n'est donc pas le seul aspect à prendre en compte, on doit également axer sur l'aspect plus humain, non pas uniquement pour les deux États, mais de voir au bien-être de la population, à la sécurité de la nation sous toutes ses formes.

Un aspect concret à prendre en compte lors des ententes est selon moi les villes de Jérusalem et de Bethleem. À ce sujet, je suggère de les traiter sous un autre angle. Comme ces deux villes sont sources de conflits depuis des décennies, pourquoi ces villes ne sont-elles pas sous tutorat international, ou ne pourraient-elle pas être un comparatif à ce qu'est le Vatican ? Un État indépendant. Dans ce cas-ci on parlerait d'un territoire regroupant ces deux villes uniquement. Ce sont des lieux saints après tout, qui pourraient se régir comme le Vatican. Elles ne seraient ni israéliennes, ni palestiniennes. Plusieurs possibilités pourraient être envisagées si la communauté internationale met du sien et décide de prendre le mandat en main. Chose certaine, on doit réagir face aux injustices et qu'on tente d'appuyer Israël ou qu'on donne notre support à la Palestine, je pense que dans tous les cas, on se doit de réagir et d'intervenir. Ces conflits et déploiements militaires tuent des milliers de civils et de gens innocents et les récents bombardements de 2014 nous ont démontré que les tensions sont loin d'être terminées et de se dissoudre. On a pu voir un non-respect des cessez-le-feu mis en branle par l'Égypte et la communauté internationale, d'un côté comme de
I'autre. Des milliers de gens vivant dans la crainte, et le désir de survie. Nous nous devons d'intervenir, et nous devons le faire rapidement. Pour le moment, les communautés locales parviennent à recevoir de l'aide d'organismes internationaux, tel Oxfam. Comme Oxfam et comme plusieurs autres d'ailleurs l'estime il est nécessaire d'en venir à un accord mutuel et une solution à deux États afin de contrer la pauvreté et les conditions de vies éprouvantes auxquelles la population fait face (Oxfam, 2015). La communauté internationale doit agir avant que d'autres vies ne soient enlevées. Des vies humaines qui comptent. Israéliennes ou Palestiniennes.

\section{Références}

BBC Mundo. (15 juillet 2014). Cómo es la vida en Gaza bajo los bombardeos y el bloqueo. $B B C$ Mundo. Repéré à http://www.bbc.co.uk/mundo/ noticias/2014/07/140715_gaza_palestinos_israel_ vida_cotidina_mz.shtml

Bourdieu. P. (1977). Annales, Économies, Sociétés, Civilisations. Persée : Revues Scientifiques, 32(3), 405-411.

Butler, J. (2010). Ce qui fait une vie. Essai sur la violence, la guerre et le deuil, Judith Butler. Paris, France : Zones.

Butler, J. (2011). Sur la relation entre la précarité et le pouvoir d'État. Conférence annuelle commémorative de I'Institut Nicos Poulantzas, Athènes, Grèce.

Crescenzi, I. (2007). Le Martyre de la Palestine, Vérités pour I'histoire. Paris, France: Dualpha Éditions.

David, C.-P. (2006). La Guerre et la Paix-Approches contemporaines de la sécurité et de la stratégie. Montréal, Québec: Presses de la fondation nationale des sciences politiques.

Debié, F. et Fouet, S. (2001). La paix en miettes Israë/ et Palestine (1993-2000). Paris, France: Presses Universitaires de France.

Éthier, D. (2007). Introduction aux relations internationales. Montréal, Canada: Les Presses de I'Université de Montréal.

Galtung, J. (1969). Violence, Peace, and Peace Research. Journal of Peace Research, 6(3), 167-191.

Gresh, A. (2007). Israël, Palestine; Vérités sur un conflit. Paris, France : Hachette Littératures.

Maltais, B. (30 novembre 2012). Genèse du conflit israélo-palestinien. Radio-Canada. Repéré 
de http://ici.radio-canada.ca/nouvelles/ international/2012/11/29/010-conflit-israelopalestinien-genese.shtml

ONU. (2006). Cartes et sources d'information géographiques. Organisation des Nations Unies. Repéré de http://www.un.org/Depts/Cartographic/ french/htmain.htm

Oxfam. (2015). Territoire occupé palestinien et Israël. Oxfam International. Repéré de https://www.oxfam. org/fr/pays/territoire-palestinien-occupe-et-israel

Saïd, E. (1994). Des intellectuels et du pouvoir. Paris, France : Seuil.

Saïd, E. (2005). L'Orientalisme: l'Orient créé par l'Occident. Paris, France : Seuil. 